\title{
New emerging drug-resistant malaria
}

\author{
This article was published in the following Dove Press journal: \\ International Journal of General Medicine \\ 7 October 2010 \\ Number of times this article has been viewed
}

\section{Viroj Wiwanitkit \\ Wiwanitkit House, Bangkhae, Bangkok Thailand \\ Date of preparation: $20^{\text {th }}$ August 2008 \\ Conflict of interest: None declared}

Clinical question: What is the best treatment for artemisinin-resistant malaria?

Results: There is still no better treatment than the presently used artemisinin-based combination therapies. A new antimalarial drug for this problem needs to be found.

Implementation: Pitfalls to avoid when treating drug-resistant malaria:

Keywords: malaria, drug resistance

\section{Drug-resistant malaria}

Definition: A "drug-resistant malaria" is a malarial infection that is not responding to the standard basic antimalarial treatment. Artemisinin is a drug used to treat multi-drug resistant strains of falciparum malaria. The World Health Organization has recommended that a switch to artemisinin combination therapies be made in all countries where the malaria parasite has developed resistance to chloroquine. Their site of action within the parasite remains controversial. At the chemical level, one theory states that when the parasite that causes malaria infects a red blood cell, it consumes hemoglobin within its digestive vacuole, liberating free heme, an iron-porphyrin complex. The iron reduces the peroxide bond in artemisinin, generating high-valency iron-oxo species and resulting in a cascade of reactions that produce reactive oxygen radicals that damage the parasite, leading to its death. Recently, emergence of new artemisinin drug-resistant malaria has been reported. This problem for falciparum malaria, of which artemisinin-based combination therapies are the recommended standard treatments, is specifically focused on herein.

Etiology: Resistant strain of malarial parasite.

Incidence: The incidence of drug-resistant malaria is different in different settings. The highest incidence is reported in Indochina (Thai-Cambodia border and ThaiMyanmar border). ${ }^{1}$

Economics: No published study has addressed the economic issues of drugresistant malaria. However, Foster et al reports that about US\$1800 million is spent annually. ${ }^{2}$

Level of evidence: Systematic reviews, meta-analyses, randomized controlled trials (RCTs).
Correspondence:Viroj Wiwanitkit Wiwanitkit House, Bangkhae, Bangkok Thailand 10160

Tel $+662-4132436$

Fax +66 2-4I32436

Emailwviroj@yahoo.com 
Search sources: PubMed, Cochrane Library, NHS Evidence, DARE, clinical evidence.

Outcomes: From the patient perspective the main outcomes are:

1. Success in antimalarial drug treatment.

2. Avoidance of complications and death.
Consumer summary: The standard classical antimalarial drugs are not fully effective against emerging strains of drug-resistant malaria. The period of taking drugs has to be extended from that normally given. The control of drug use and surveillance of drug-resistant strains are the present means to fight this problem. New antimalarial drugs to solve the forthcoming problem are required.

\section{The evidence}

\section{How does artemisinin-resistant malaria affect the present standard antimalarial treatment?}

Systematic reviews: 1

Meta-analyses: 3

RCTs: 1

There is a systematic review on artemisinin-resistant falciparum malaria. ${ }^{3}$ Partial artemisinin resistance is the present problem. ${ }^{3}$ The emerging problem is proposed to be due to selection of the resistant phenotype which is the result of exposure of the parasite population to artemisinin monotherapies in subtherapeutic doses for over 30 years, and the availability of substandard artemisinins. ${ }^{3}$ Focusing on the meta-analyses, for non-artemisinin-resistant cases, it is noted that "The addition of 3 days of artesunate to standard antimalarial treatments substantially reduces treatment failure, recrudescence, and gametocyte carriage." Similar notes are reported in the other two meta-analyses. 5,6 However, there is no specific meta-analysis on artemisininresistant falciparum malaria. The randomized trial concluded "Resistance is characterized by slow parasite clearance in vivo without corresponding reductions on conventional in vitro susceptibility testing."’

\section{The practice}

\section{Potential pitfalls}

Entering the endemic areas without concern for the existence of new drug-resistant malaria might lead to a lack of prevention. A history of exposure is required for early diagnosis and adjustment for the longer artemisinin-based combination therapies.

\section{Management}

Artemisinin-resistant malaria should not be managed by non-specialists. Suspected cases should be referred to specialists in infectious diseases or tropical medicine.

\section{Which treatments are best for cases of drug-resistant malaria?}

Systematic reviews: 0

Meta-analyses: 0

RCTs: 1

There are no systematic reviews or meta-analyses for artemisinin-resistant falciparum malaria. The randomized trial showed that the present artemisinin-based combination therapies are still successful treatments but the clearance of malaria took longer than for the non-resistant cases. ${ }^{7}$

Prolonged drug administration is required and there is no report of adverse drug reaction due to this extended drug administration. $^{7}$

\section{Conclusion}

There is evidence on emergence of artemisinin-resistant malaria from highly endemic areas in Southeast Asia. Longer administration of standard artemisinin-based combination therapies is required for treatment of drug-resistant malaria. If resistance is complete, failure of present artemisinin-based combination therapies can be expected, and development of a new antimalarial drug for artemisinin-resistant malaria is required.

\section{Assessment}

- Traveling or living in the endemic areas (Indochina, Southeast Asia) poses a high risk.

- If history suggests a possibility of exposure and the diagnosis confirms falciparum malaria, refer to a specialist.

- If the primary standard treatment does not clear malaria, reassessment of patient history is needed and referral to a specialist is suggested.

\section{Treatment}

- Treatment must be by a specialist. 
- Prolonged artemesin treatment is the present standard recommendation and there is no suggested alternative treatment.

- Long artemisinin-based combination treatment until clearance of malaria.

- Close monitoring of clinical appearance and parasite clearance during treatment.

- Reporting to the local disease control authority is required.

\section{Indications for specialist referral}

- A history of visiting endemic areas.

- Failure of primary standard artemisinin-based combination treatments.

\section{Further reading}

Csizmadia E, Kalnoky I. Antimalarial Drugs: Costs, Safety and Efficacy. New York, NY: Nova Publishers; 2009.

Li Q, Milhous WK, Weina PJ. Artemisinins in Malaria Therapy. New York, NY: Nova Publishers; 2007.
Wiwanitkit V. Malaria Research in Southeast Asia. New York: Nova Publishers; 2007.

\section{References}

1. Wongsrichanalai C, Sirichaisinthop J, Karwacki JJ, et al. Drug resistant malaria on the Thai-Myanmar and Thai-Cambodian borders. Southeast Asian J Trop Med Public Health. 2001;32:41-49.

2. Foster S, Phillips M. Economics and its contribution to the fight against malaria. Ann Trop Med Parasitol. 1998;92:391-398.

3. Dondorp AM, Yeung S, White L, Nguon C, Day NPJ, Socheat D, von Seidlein L. Artemisinin resistance: current status and scenarios for containment Nature Rev Microbiol. 2010;8:272-280.

4. Babiker A, Garner P, et al. Artesunate combinations for treatment of malaria: meta-analysis. Lancet. 2004;363:9-17.

5. Obonyo CO, Juma EA, Ogutu BR, et al. Amodiaquine combined with sulfadoxine/pyrimethamine versus artemisinin-based combinations for the treatment of uncomplicated falciparum malaria in Africa: a metaanalysis. Trans R Soc Trop Med Hyg. 2007;101:117-126.

6. Bukirwa H, Critchley J. Sulfadoxine-pyrimethamine plus artesunate versus sulfadoxine-pyrimethamine plus amodiaquine for treating uncomplicated malaria. Cochrane Database Syst Rev. 2006;1:CD004966.

7. Dondorp AM, Nosten F, Yi P, et al. Artemisinin resistance in Plasmodium falciparum malaria. $N$ Engl J Med. 2009;361:455-467.
International Journal of General Medicine

\section{Publish your work in this journal}

The International Journal of General Medicine is an international peer-reviewed open-access journal that focuses on general and internal medicine, pathogenesis, epidemiology, diagnosis, monitoring and treatment protocols. The journal is characterized by the rapid reporting of reviews, original research and clinical studies across all disease areas.

\section{Dovepress}

A key focus is the elucidation of disease processes and management protocols resulting in improved outcomes for the patient. The manuscript management system is completely online and includes a very quick and fair peer-review system. Visit http://www.dovepress.com/ testimonials.php to read real quotes from published authors. 